\title{
Delayed onset of lactogenesis and reduced breastfeeding frequency in mothers who give birth by caesarean section
}

\author{
Niamh M. Kelly ${ }^{1}$, Jennifer T. Smilowitz ${ }^{2}$, Orla Cagney ${ }^{3}$, Robin L. Flannery ${ }^{3}$ and \\ Rachel M Tribe \\ ${ }^{1}$ Division of Women's Health, King's College London, St Thomas' Hospital, London, United Kingdom, \\ ${ }^{2} U C$ Davis, California, USA and \\ ${ }^{3}$ Evolve BioSystems Inc., California, USA
}

\begin{abstract}
Lactogenesis stage II, also known as when a mother's milk "comes in", is characterised by copious milk production. Delayed lactogenesis II, when onset occurs after 72 hours post-partum, has been linked to early breastfeeding cessation. It has been suggested that caesarean section is a risk factor for late onset of lactogenesis II. It is unknown why lactogenesis II may be delayed in caesarean section but there are several potential reasons such as volume of blood loss, maternal stress, delayed breastfeeding initiation and difficulties with mobility and positioning. Analysis of timing of lactogenesis and breastfeeding frequency was carried out on data from the PROMESA and IMPRINT studies, which were looking at the supplementation of breast milk with a probiotic Bifidobacterium infantis. IMPRINT was carried out in California and enrolled eighty women prior to birth or before postnatal day 4 . The PROMESA study in the UK only recruited women who were booked for elective caesarean sections, and also enrolled eighty mother-baby dyads. As part of both studies mothers filled out a variety of surveys and daily logs, including a daily feeding log, along with selfreported lactogenesis. Using logistic regression, we looked at whether mode of birth (spontaneous vaginal delivery, emergency and elective caesarean section) was associated with the timing of onset of lactogenesis, and linear regression to look at the difference in breastfeeding frequency between modes of birth. Mode of birth was significantly associated with delayed onset of lactogenesis $>3$ days (OR 3.38, 95\% CI 2.48-4.61). There was also a reduced frequency of breastfeeding in the first week post-partum in motherbaby dyads who underwent an elective caesarean section. These findings suggest that mothers who give birth by elective caesarean section may need additional support with breastfeeding in the early days post-partum, as well as ongoing support long-term to reduce the likelihood of early cessation of breastfeeding.
\end{abstract}

\section{Conflict of Interest}

Funding: Evolve BioSystems, Inc. sponsored and funded the PROMESA and IMPRINT studies. Paul Seed and Rachel Tribe are funded by Tommy's charity, NIHR Biomedical Research Centre at Guy's and St Thomas' NHS Foundation Trust and King's College London. 